Access flow 89

Acetate-free biofiltration 166

Acute kidney failure 79

- _ injury 79, 120, 214

- lung injury 44

- renal failure 195

- respiratory failure 44

Adiponectin 1

Adsorption 34

Amino acid profile 166

Anemia 186

Ankle-brachial index 50

Antibacterial agents 195

Anticoagulation 79

Arm muscle area 161

Atherosclerosis 277

-, survival 272

Blood temperature monitoring 89

Body mass index 161

- surface area formulae estimate 296

Calcium 111

Cardiovascular disease 50

CCN2 272

CERA 186

Charcoal 84

Charlson comorbidity index 16

Chronic kidney disease 16, 186, 277

Clinical features 172

- targets 111

Comorbidity 56

Connective tissue growth factor 272

Continuous hemofiltration 79

- renal replacement therapy 37, 79, 195

C-reactive protein 8,50

Critical care nephrology 214

Cytokine adsorption 44

Cytokine-adsorbing column 44

Dalbavancin 37

Darbepoetin 186

Depression 98

Dialysate 71

Dialysis 272

- dose prescription 214

- prescription 296

-, reinitiation 56

Diet 71

Disability, physical 98

Disinfection 64
Endotoxin 44

End-stage renal disease 50, 178, 266

Environment 181

Eptifibatide 266

Equivalent continuous clearance 257

- renal clearance 257

Extracorporeal circuit 79

Financial savings 181

Fluid overload 71

Fractional solute removal 257

Free immunoglobulin light chains 34

Genetic polymorphism 272

Glomerular filtration rate equations 296

Guanidino compounds 277

H1N1 virus infection 172

Haemodialysis 111

Health-related quality of life 98

Heart failure 146

Hemodiafiltration 25, 34

- with endogenous reinfusion 34, 166

Hemodialysis 37, 64, 71, 98, 135, 181, 266

- treatment time 8

Hemoglobin 186

Hemoperfusion 84

Hospital cost 120

Hospitalization time 146

Hypertension 71, 288

Hypotension, intradialytic 71

Inflammation 127

Infusion flow 25

Intoxication 84

Kidney Disease Outcomes Quality Initiative 111

- failure 56

Kinetic modeling 257

Long-term hemodialysis 161

Macroporous resin 34

Malnutrition-inflammation complex syndrome 16

- score 16

Massive gastrointestinal hemorrhage 178

Metabolic syndrome 1

Mid-dilution 25

Mortality 120, 127

-, risk factors 56
Ozone 64

Parathyroid hormone 111

Peritoneal dialysis $1,16,127,146$

- transport rate 127

Pharmacodynamics 195

Pharmacokinetics 195, 266

Phosphate 111

Protein clearance, peritoneal 127

Renal dialysis 172

- disease 288

- failure 146

- replacement therapy 214

Reverse osmosis 181

\section{SDMA 277}

Serum albumin 8

- creatinine 120

Sodium profiling 71

Solute clearance 37

- removal 25

- - index 257

Sudden death 135

Taiwan 98

Toxicology 84

Transient loss of consciousness 178

Transmembrane pressure 25

Transplantation, kidney 56

Ultrafiltration rate 8

Ultrasound 89

Uremic toxins 34, 277

Uric acid 288

Vascular damage 277

Volume control 50

- overload 127

Water conservation 181

- reuse 181

- treatment 64 